\title{
Novel Criteria of ISS Analysis for Delayed Memristive Simplified Cohen-Grossberg BAM Neural Networks
}

\author{
Yong Zhao $\mathbb{D}$ and Shanshan Ren \\ School of Mathematics and Information Science, Henan Polytechnic University, Jiaozuo 454000, China \\ Correspondence should be addressed to Yong Zhao; zhaoyong_54@163.com
}

Received 30 September 2019; Accepted 18 December 2019; Published 24 January 2020

Academic Editor: Sing Kiong Nguang

Copyright (C) 2020 Yong Zhao and Shanshan Ren. This is an open access article distributed under the Creative Commons Attribution License, which permits unrestricted use, distribution, and reproduction in any medium, provided the original work is properly cited.

\begin{abstract}
The memristor as the fourth circuit element, it can capture some key aspects of biological synaptic plasticity. So, it is significant that the characteristic of memristors is considered in neural networks. This paper investigates input-to-state stability (ISS) of a class of memristive simplified Cohen-Grossberg bidirectional associative memory (BAM) neural networks with variable time delays. In the sense of Filippov solution, some novel sufficient criteria for ISS are obtained based on differential inclusions and differential inequalities; when the input is zero, the stability of the total system is state stable. Furthermore, numerical simulations are illustrated to show the feasibility of our results.
\end{abstract}

\section{Introduction}

In 1988, Kosko proposed a class of bidirectional associative memory (BAM) neural networks [1]. Because of potential applications of associate memory and pattern recognition, many research studies are increasingly concerned about dynamics behaviors of such neural networks. There have been many results on the stability for BAM neural networks with or without delays [2-8]. At the same time, in 1983, Cohen and Grossberg discussed a class of competitive neural networks, which is called Cohen-Grossberg neural networks. A lot of models can be described by this model, such as Hopfield-type neural networks, population biology. Meanwhile, this model has also been applied in some areas such as associate memory, pattern recognition, and optimization problems. Therefore, stability problems have attracted many researchers'attentions [9-16]. Based on nonsmooth analysis and stability theory, we obtained sufficient conditions of the globally asymptotical stability of the mix-delayed Cohen-Grossberg neural networks with nonlinear impulse [13]. Considering characteristics of BAM neural networks and Cohen-Grossberg neural networks, many researchers investigated qualitative analysis of Cohen-Grossberg BAM neural networks [16-18]. Bao [16] investigated the existence and exponential stability of periodic solutions for fuzzy Cohen-Grossberg BAM neural networks with mixed delays by M-matrix theory and inequality techniques. Rao et al. [17] considered stability results for p-Laplace dynamical equations including Cohen-Grossberg BAM neural networks by constructing suitable Lyapunov functional, M-matrix technique, and Yang inequality. Chinnathambi et al. [18] explored finitetime stabilization of delayed Cohen-Grossberg BAM neural networks under suitable control schemes by using Lyapunov function and some algebraic conditions.

In 1971, Chua predicted memristors as the fourth circuit element [19]. Strukov et al. found the first practical memristor device in 2008 [20]. It can remember its past dynamic history, similar to biological synapse. Because of the characteristic of its memory, it attracted some theoretical researchers' interests and notices [21-25]. The stability of neural networks with memristors has become a hot topic [24-29]. Considering the characteristic of memristors is significant in neural networks, it can give a potential hope to build brain-like computer and emulate human brain.

In practice, information transmission unavoidably exists in delays. In neural networks, finite propagation velocity often results in delays. Delayed neural network reduces to 
more complex dynamical behaviors, such as periodic, instability, and chaos. Thus, considering time delays is significant in neural networks [2-14, 16, 18, 24-34]. Recently, dynamics analysis for memristive neural networks with delays has attracted considerable attention [21-34]. Wu and Zeng [27] investigated the Lagrange stability of memristive neural networks by the nonsmooth analysis and control theory, which is considered to discuss the stability of the total system and not only the stability of the equilibria. Zhao et al. [28] considered the input-to-state stability of a class of memristive Cohen-Grossberg-type neural networks with variable time delays, which include some known results as particular cases. Zhong et al. [29] obtained some sufficient conditions for the input-to-state stability of a class of memristive neural networks with time-varying delays. Furthermore, Zhao et al. [30] discussed dynamics of memristive BAM neural networks with variable time delays and obtained some novel sufficient conditions of the inputto-state stability.

From these papers, we know the input-to-state stability is general stability. When the inputs are bounded, it is also called bounded-input bounded-output (BIBO) stable. When the inputs are zero, the state of the system is asymptotically stable. Therefore, considering input-to-state stability of system (1) is necessary and significant. Thus, inspired by the above discussions, we will here investigate stability problems of memristive simplified Cohen-Grossberg BAM neural networks with variable time delays:

$$
\left\{\begin{array}{r}
\dot{x}_{i}(t)=c_{i}\left(x_{i}(t)\right)\left[-x_{i}(t)+\sum_{j=1}^{m} a_{j i}\left(x_{i}(t)\right) f_{j}\left(y_{j}(t)\right)+\sum_{j=1}^{m} b_{j i}\left(x_{i}(t)\right) f_{j}\left(y_{j}(t-\tau(t))\right)+I_{i}(t)\right] \\
\dot{y}_{j}(t)=d_{i}\left(y_{j}(t)\right)\left[-y_{j}(t)+\sum_{i=1}^{n} c_{i j}\left(y_{j}(t)\right) g_{i}\left(x_{i}(t)\right)+\sum_{i=1}^{n} d_{i j}\left(y_{j}(t)\right) g_{i}\left(x_{i}(t-\tau(t))\right)+J_{j}(t)\right] \\
t \geq 0, i=1,2, \ldots, n ; j=1,2, \ldots, m,
\end{array}\right.
$$

where $c_{i}(\cdot)$ and $d_{i}(\cdot)$ are the amplification functions, $f_{j}(j=1,2, \ldots, m)$ and $g_{i}(i=1,2, \ldots, n)$ denote the signal transmission functions, and $I_{i}(t)(i=1,2, \ldots, n)$ and $J_{j}(t)(j=1,2, \ldots, m)$ are the external inputs. The delays $\tau(t) \geq 0 \quad$ satisfy $\quad 0<\tau(t)<\tau, \dot{\tau}(t) \leq \mu<1 . \quad a_{j i}\left(x_{i}(t)\right)$, $b_{j i}\left(x_{i}(t)\right), c_{i j}\left(y_{j}(t)\right)$, and $d_{i j}\left(y_{j}(t)\right)$ represent memristorbased weights, and

$$
\begin{aligned}
& a_{j i}\left(x_{i}(t)\right)=\frac{W_{j i}}{C_{i}} \times \operatorname{sgn}_{i j}, \\
& b_{j i}\left(x_{i}(t)\right)=\frac{M_{j i}}{C_{i}} \times \operatorname{sgn}_{i j}, \\
& \operatorname{sgn}_{i j}= \begin{cases}1, & i \neq j, \\
-1, & i=j,\end{cases} \\
& c_{i j}\left(y_{j}(t)\right)=\frac{W_{i j}}{C_{j}} \times \operatorname{sgn}_{i j}, \\
& d_{i j}\left(y_{j}(t)\right)=\frac{M_{i j}}{C_{j}} \times \operatorname{sgn}_{i j}, \\
& \operatorname{sgn}_{i j}= \begin{cases}1, & i \neq j, \\
-1, & i=j .\end{cases}
\end{aligned}
$$

According to the feature of a memristor and its currentvoltage characteristic, we have

$$
\begin{aligned}
& a_{j i}\left(x_{i}(t)\right)= \begin{cases}\widehat{a}_{j i}, & \operatorname{sgn}_{j i} \frac{\mathrm{d} f_{j}\left(y_{j}(t)\right)}{\mathrm{d} t}-\frac{\mathrm{d} x_{i}(t)}{\mathrm{d} t} \leq 0, \\
\check{a}_{j i}, & \operatorname{sgn}_{j i} \frac{\mathrm{d} f_{j}\left(y_{j}(t)\right)}{\mathrm{d} t}-\frac{\mathrm{d} x_{i}(t)}{\mathrm{d} t}>0,\end{cases} \\
& b_{j i}\left(x_{i}(t)\right)= \begin{cases}\widehat{b}_{j i}, & \operatorname{sgn}_{j i} \frac{\mathrm{d} f_{j}\left(y_{j}(t-\tau(t))\right)}{\mathrm{d} t}-\frac{\mathrm{d} x_{i}(t)}{\mathrm{d} t} \leq 0, \\
\check{b}_{j i}, & \operatorname{sgn}_{j i} \frac{\mathrm{d} f_{j}\left(x_{j}(t-\tau(t))\right)}{\mathrm{d} t}-\frac{\mathrm{d} x_{i}(t)}{\mathrm{d} t}>0,\end{cases} \\
& c_{i j}\left(y_{j}(t)\right)= \begin{cases}\widehat{c}_{i j}, & \operatorname{sgn}_{i j} \frac{\mathrm{d} g_{i}\left(x_{i}(t)\right)}{\mathrm{d} t}-\frac{\mathrm{d} y_{j}(t)}{\mathrm{d} t} \leq 0, \\
\check{c}_{i j}, & \operatorname{sgn}_{i j} \frac{\mathrm{d} g_{i}\left(x_{i}(t)\right)}{\mathrm{d} t}-\frac{\mathrm{d} y_{j}(t)}{\mathrm{d} t}>0, \\
\check{d}_{i j}, & \operatorname{sgn}_{i j} \frac{\mathrm{d} g_{i}\left(x_{i}(t-\tau(t))\right)}{\mathrm{d} t}-\frac{\mathrm{d} y_{j}(t)}{\mathrm{d} t}>0 .\end{cases} \\
& d_{i j}\left(y_{j}(t)\right)= \begin{cases}\widehat{d}_{i j}, & \operatorname{sgn}_{i j} \frac{\mathrm{d} g_{i}\left(x_{i}(t-\tau(t))\right)}{d t}-\frac{\mathrm{d} y_{j}(t)}{\mathrm{d} t} \leq 0, \\
& \end{cases}
\end{aligned}
$$

This system includes some well-known systems as particular cases. The solution of system (1) is represented by $(x(t), y(t))^{T}=\left(x_{1}(t), \ldots, x_{n}(t), y_{1}(t), \ldots, y_{m}(t)\right)^{T} \in$ $R^{n+m}$.

System (1) is supplemented with the initial values 


$$
\begin{aligned}
& x_{i}(s)=\varphi_{i}(s), \quad s \in(-\tau, 0], i=1,2, \ldots, n, \\
& y_{j}(s)=\psi_{j}(s), \quad s \in(-\tau, 0], j=1,2, \ldots, m,
\end{aligned}
$$

where $\varphi_{i}(s), \psi_{j}(s)$ denotes a real-valued continuous function defined on $(-\tau, 0]$.

To obtain our solutions of system (1), furthermore, we assume as follows:

(H1) $\quad c_{i}(\cdot): R \longrightarrow R$ is positive, continuous, and bounded such that $0<\underline{c}_{i} \leq c_{i}(\cdot) \leq \bar{c}_{i}<\infty$.

(H2) $d_{i}(\cdot): R \longrightarrow R$ is positive, continuous, and bounded such that $0<\underline{d}_{i} \leq d_{i}(\cdot) \leq \bar{d}_{i}<\infty$.

$(H 3) f_{j}(\cdot)$ and $g_{i}(\cdot)$ satisfy globally Lipschitz conditions with positive constants $k_{j}>0$ and $l_{i}>0$, respectively, such that

$$
\begin{gathered}
\left|f_{j}(x)-f_{j}(y)\right| \leq k_{j}|x-y|, \\
\left|g_{i}(x)-g_{i}(y)\right| \leq l_{i}|x-y|,
\end{gathered}
$$

for any $x(t), y(t) \in R$.

The rest of this paper is organized as follows. In Section 2 , we introduce some notations, definitions, and some preliminary results. In Section 3, we present sufficient conditions for the input-to-state stability of system (1). Finally, in Section 4, an example illustrates the feasibility of our results.

\section{Preliminaries}

In this section, we give some notations, definitions, and some preliminaries, which will be necessary for our main results.

In this paper, the solutions of all systems are considered in Filippov's sense [35]. $\operatorname{co}\{\widehat{a}, \breve{a}\}$ denotes the closure of the convex hull generated by the real numbers $\hat{a}$ and $\check{a}$. $x(t)=\left(x_{1}(t), \ldots, x_{n}(t)\right)^{T} \in R^{n}$ denotes a column vector, $\|x\|=\max _{1 \leq i \leq n}\left\{\left|x_{i}\right|\right\},|x|=\left(\left|x_{1}\right|, \ldots,\left|x_{n}\right|\right)^{T} ; \quad I(t) \in L_{\infty}^{n}$ : $R^{+} \longrightarrow R^{n}$ with $\|I\|_{\text {sup }}=\sup \{\|I(t)\|, t \geq 0\}<+\infty ; J(t) \epsilon$ $L_{\infty}^{n}: R^{+} \longrightarrow R^{n}$ with $\|I\|_{\text {sup }}=\sup \{\|I(t)\|, t \geq 0\}<+\infty$. Let $\tilde{a}_{j i}=\max \left\{\left|\widehat{a}_{j i}\right|,\left|\check{a}_{j i}\right|\right\}, \widetilde{b}_{j i}=\max \left\{\left|\widehat{b}_{j i}\right|,\left|\check{b}_{j i}\right|\right\}, \quad$ for $\quad i, j=$ $1,2, \ldots, n$.

In what follows, we also need to introduce some definitions:

Definition 1. Let $E \subset R^{n}, x \mapsto F(x)$ be called a set-valued map from $E \hookrightarrow R^{n}$, if for $x \in E$, there is a corresponding nonempty set $F(x) \subset R^{n}$.
Definition 2. For the system $\mathrm{d} x / \mathrm{d} t=g(x), x \in R^{n}$, with discontinous right-hand sides, a set-valued map is defined as

$$
\phi(x)=\bigcap_{\delta>0} \bigcup_{\mu(N)=0} \overline{\operatorname{co}}\left[\frac{g(B(x, \delta))}{N}\right],
$$

where $\overline{\mathrm{co}}[E]$ is the closure of the convex hull of the set $E$, $B(x, \delta)=\{y: y-x \leq \delta\}$, and $\mu(N)$ is a Lebesgue measure of the set $N$. A solution in Filippov's sense of the Cauchy problem for this system with initial condition $x(0)=x_{0}$ is an absolutely continuous function $x(t), t \in[0, T]$, which satisfies $x(0)=x_{0}$ and the differential inclusion [36]

$$
\frac{\mathrm{d} x}{\mathrm{~d} t} \in \phi(x), \quad \text { for a.e. } T \in[0, T] \text {. }
$$

Definition 3 (see [29, 37]). A scalar continuous function $\alpha(r)$ defined for $r \in[0, a)$ is said to belong to the class $\kappa$ if it is strictly increasing, and $\alpha(0)=0$. It is said to belong to the class $\kappa_{\infty}$ for all $r \geq 0$ and also $\alpha(r) \longrightarrow+\infty$ as $r \longrightarrow+\infty$.

Definition 4 (see $[29,37])$. A function $\beta(r, s)$, defined for $r \in[0, a)$ and $s \in[0, \infty)$ is said to belong to the class $\kappa L$ if for each fixed $s \geq 0$, the mapping $\beta(r, s)$ belongs to the class $K$ with respect to $r$ and for each fixed $r$, and the mapping $\beta(r, s)$ is decreasing with respect to $s$ and $\beta(r, s) \longrightarrow 0$ as $s \longrightarrow \infty$.

Definition 5 (see $[29,37]$ ). System (1) is said to be input-tostate stable if there exists a $\kappa L$ function $\beta$ and a $\kappa_{\infty}$ function $\alpha$ such that

$$
\begin{aligned}
\left\|x\left(t ; x_{0}, I(t)\right)\right\|+\left\|y\left(t ; y_{0}, J(t)\right)\right\| \leq & \beta\left(\left\|x_{0}\right\|_{\infty}+\left\|y_{0}\right\|_{\infty}, t\right) \\
& +\alpha\left(\|I(t)\|_{\text {sup }},\|J(t)\|_{\text {sup }}\right), \quad t \geq 0,
\end{aligned}
$$

for any $x_{0} \in R^{n}, y_{0} \in R^{m}, I(t), J(t) \in L_{\infty}^{n}$.

Remark 1. When the inputs $I(t)$ and $J(t)$ are bounded, note that $\beta$ is a $\kappa_{\infty}$ function and is also bounded. When the inputs $I(t)$ and $J(t)$ are zero, system (1) is asymptotically stable. From (8), $\left\|x\left(t ; x_{0}, I(t)\right)\right\|+\left\|y\left(t ; y_{0}, J(t)\right)\right\|$ is bounded. Therefore, system (1) is input-to-state stable and also called bounded-input bounded-output (BIBO) stable.

\section{Main Results}

We will now discuss the input-to-state stability of system (1) based on differential inclusions and set-valued maps [30, 31]. System (1) can be rewritten as follows:

$$
\begin{aligned}
& \dot{x}_{i}(t) \in c_{i}\left(x_{i}(t)\right)\left[-x_{i}(t)+\sum_{j=1}^{m} \operatorname{co}\left(\widehat{a}_{j i}, \check{a}_{j i}\right) f_{j}\left(y_{j}(t)\right)+\sum_{j=1}^{m} \operatorname{co}\left(\widehat{b}_{j i}, \check{b}_{j i}\right) f_{j}\left(y_{j}(t-\tau(t))\right)+I_{i}(t)\right], \quad t \geq 0, i=1,2, \ldots, n, \\
& \dot{y}_{j}(t) \in d_{j}\left(y_{j}(t)\right)\left[-y_{j}(t)+\sum_{i=1}^{n} \operatorname{co}\left(\widehat{c}_{i j}, \check{c}_{i j}\right) g_{i}\left(x_{i}(t)\right)+\sum_{i=1}^{n} \operatorname{co}\left(\widehat{d}_{i j}, \check{d}_{i j}\right) g_{i}\left(x_{i}(t-\tau(t))\right)+J_{j}(t)\right], \quad t \geq 0, j=1,2, \ldots, m .
\end{aligned}
$$


Equivalently, for $i=1,2, \ldots, n$ and $j=1,2, \ldots, m$, there $\operatorname{exist} \Phi_{j i} \in \operatorname{co}\left(\widehat{a}_{j i}, \check{a}_{j i}\right)$ and $\Psi_{j i} \in \operatorname{co}\left(\widehat{b}_{j i}, \check{b}_{j i}\right), \Theta_{i j} \in \operatorname{co}\left(\widehat{c}_{i j}, \check{c}_{i j}\right)$ and $\Lambda_{i j} \in \operatorname{co}\left(\hat{d}_{i j}, \check{d}_{i j}\right)$ such that

$$
\begin{aligned}
& \dot{x}_{i}(t)=c_{i}\left(x_{i}(t)\right)\left[-x_{i}(t)+\sum_{j=1}^{m} \Phi_{j i} f_{j}\left(y_{j}(t)\right)+\sum_{j=1}^{m} \Psi_{j i} f_{j}\left(y_{j}(t-\tau(t))\right)+I_{i}(t)\right], \quad t \geq 0, i=1,2, \ldots, n, \\
& \dot{y}_{j}(t)=d_{j}\left(y_{j}(t)\right)\left[-y_{j}(t)+\sum_{i=1}^{n} \Theta_{i j} g_{i}\left(x_{i}(t)\right)+\sum_{i=1}^{n} \Lambda_{i j} g_{i}\left(x_{i}(t-\tau(t))\right)+J_{j}(t)\right], \quad t \geq 0, j=1,2, \ldots, m .
\end{aligned}
$$

The above parameters $\Phi_{j i}, \quad \Psi_{j i}, \Theta_{i j}$, and $\Lambda_{i j}$ $(i=1,2, \ldots, n$ and $j=1,2, \ldots, m)$ in system (10) depend on the initial conditions of system (1) and time $t$. Clearly, in Filippov's sense, the solution of system (1) with initial values given by

$$
\begin{aligned}
& x_{i}(s)=\varphi_{i}(s), \quad s \in(-\tau, 0], i=1,2, \ldots, n, \\
& y_{j}(s)=\psi_{j}(s), \quad s \in(-\tau, 0], j=1,2, \ldots, m,
\end{aligned}
$$

is absolutely continuous on any compact interval of $[0,+\infty]$. Therefore,

$$
\begin{aligned}
& \dot{x}_{i}(t) \in c_{i}\left(x_{i}(t)\right)\left[-x_{i}(t)+\sum_{j=1}^{m} \operatorname{co}\left(\widehat{a}_{j i}, \check{a}_{j i}\right) f_{j}\left(y_{j}(t)\right)+\sum_{j=1}^{m} \operatorname{co}\left(\widehat{b}_{j i}, \check{b}_{j i}\right) f_{j}\left(y_{j}(t-\tau(t))\right)+I_{i}(t)\right], \quad t \geq 0, i=1,2, \ldots, n, \\
& \dot{y}_{j}(t) \in d_{j}\left(y_{j}(t)\right)\left[-y_{j}(t)+\sum_{i=1}^{n} \operatorname{co}\left(\widehat{c}_{i j}, \check{c}_{i j}\right) g_{i}\left(x_{i}(t)\right)+\sum_{i=1}^{n} \operatorname{co}\left(\widehat{d}_{i j}, \check{d}_{i j}\right) g_{i}\left(x_{i}(t-\tau(t))\right)+J_{j}(t)\right], \quad t \geq 0, j=1,2, \ldots, m .
\end{aligned}
$$

It is obvious that for $i=1,2, \ldots, n$ and $j=1,2, \ldots, m$, the set-valued map

$$
\begin{aligned}
& x_{i}(t) \longrightarrow c_{i}\left(x_{i}(t)\right)\left[-x_{i}(t)+\sum_{j=1}^{n} \operatorname{co}\left(\widehat{a}_{j i}, \check{a}_{j i}\right) f_{j}\left(y_{j}(t)\right)+\sum_{j=1}^{n} \operatorname{co}\left(\widehat{b}_{j i}, \check{b}_{j i}\right) f_{j}\left(y_{j}(t-\tau(t))\right)+I_{i}(t)\right], \quad t \geq 0, i=1,2, \ldots, n, \\
& y_{j}(t) \longrightarrow d_{j}\left(y_{j}(t)\right)\left[-y_{j}(t)+\sum_{i=1}^{n} \operatorname{co}\left(\widehat{c}_{i j}, \check{c}_{i j}\right) g_{i}\left(x_{i}(t)\right)+\sum_{i=1}^{n} \operatorname{co}\left(\widehat{d}_{i j}, \check{d}_{i j}\right) g_{i}\left(x_{i}(t-\tau(t))\right)+J_{j}(t)\right], \quad t \geq 0, j=1,2, \ldots, m,
\end{aligned}
$$

has nonempty compact convex values. Furthermore, it is upper semicontinuous. Thus, the solution $(x(t), y(t))^{T}$ in system (10) with initial values (11) can exist in Filippov's sense, refer to [35].
Theorem 1. Assume that (H1)-(H3) hold. Then, system (1) is input-to-state stable if

$$
\begin{gathered}
\bar{c}_{i}-2 \underline{c}_{i}+\sum_{j=1}^{m}\left(\tilde{a}_{j i}+\widetilde{b}_{j i}\right) k_{j} \bar{c}_{i}+\sum_{j=1}^{m}\left(\tilde{c}_{i j}+\frac{\widetilde{d}_{i j}}{1-\mu}\right) l_{i} \bar{d}_{j}<0, \quad i=1,2, \ldots, n, \\
\bar{d}_{j}-2 \underline{d}_{j}+\sum_{i=1}^{n}\left(\widetilde{c}_{i j}+\widetilde{d}_{i j}\right) l_{i} \bar{d}_{j}+\sum_{i=1}^{n}\left(\tilde{a}_{j i}+\frac{\widetilde{b}_{j i}}{1-\mu}\right) k_{j} \bar{c}_{i}<0, \quad j=1,2, \ldots, m .
\end{gathered}
$$


Proof. Taking (14), we choose a sufficiently small positive constant $\varepsilon>0$ such that

$$
\begin{gathered}
\varepsilon+\bar{c}_{i}-2 \underline{c}_{i}+\sum_{j=1}^{m}\left(\widetilde{a_{j i}}+\widetilde{b_{j i}}\right) k_{j} \bar{c}_{i}+\sum_{j=1}^{m}\left(\widetilde{c_{i j}}+\frac{\widetilde{d_{i j}}}{1-\mu}\right) l_{i} \bar{d}_{j}<0, \quad i=1,2, \ldots, n, \\
\varepsilon+\bar{d}_{j}-2 \underline{d}_{j}+\sum_{i=1}^{n}\left(\widetilde{c_{i j}}+\widetilde{d_{i j}}\right) l_{i} \bar{d}_{j}+\sum_{i=1}^{n}\left(\widetilde{a_{j i}}+\frac{\widetilde{b_{j i}}}{1-\mu}\right) k_{j} \bar{c}_{i}<0, \quad j=1,2, \ldots, m,
\end{gathered}
$$

and consider Lyapunov function

$$
V(x(t), y(t), t)=e^{\varepsilon t}\left(x^{T}(t) x(t)+y^{T}(t) y(t)\right),
$$

where $\quad x(t)=\quad\left(x_{1}(t), x_{2}(t), \ldots, x_{n}(t)\right)^{T}, y(t)=$ $\left(y_{1}(t), y_{2}(t), \ldots, y_{m}(t)\right)^{T}$. Then,

$$
\begin{aligned}
& V(x(t), y(t), t) \\
& =V(x(0), y(0), 0)+\int_{0}^{t} \varepsilon V(x(s), y(s), s) d s+\int_{0}^{t} 2 e^{\varepsilon s}\left(x^{T}(s) \dot{x}(s)+y^{T}(s) \dot{y}(s)\right) d s \\
& \leq V(x(0), y(0), 0)+\int_{0}^{t} \varepsilon V(x(s), y(s), s) d s+\int_{0}^{t} 2 e^{\varepsilon s}\left\{\sum _ { i = 1 } ^ { n } \left[-\underline{c}_{i} x_{i}^{2}(s)+\sum_{j=1}^{m} \bar{c}_{i} \widetilde{a_{j i}} k_{j}\left|x_{i}(s)\right|\left|y_{j}(s)\right|\right.\right. \\
& +\sum_{j=1}^{m} \bar{c}_{i} \widetilde{b}_{j i} k_{j}\left|x_{i}(s)\right|\left|y_{j}(s-\tau(s))\right|+\bar{c}_{i}\left|x_{i}(s)\right|\left|I_{i}(s)\right|+\sum_{j=1}^{m}\left[-\underline{d}_{j} y_{j}^{2}(s)+\sum_{i=1}^{n} \bar{d}_{j} c_{i j} l_{i}\left|x_{i} \widetilde{(s)}\right|\left|y_{j}(s)\right|\right. \\
& \left.\left.+\sum_{i=1}^{n} \bar{d}_{j} \widetilde{d_{j i}} l_{i}\left|y_{j}(s)\right|\left|x_{i}(s-\tau(s))\right|+\bar{d}_{j}\left|y_{j}(s)\right|\left|J_{j}(s)\right|\right]\right\} \mathrm{d} s \\
& \leq V(x(0), y(0), 0)+\int_{0}^{t} \varepsilon V(x(s), y(s), s) \mathrm{d} s+\int_{0}^{t} e^{\varepsilon s}\left\{\sum _ { i = 1 } ^ { n } \left[-2 \underline{c}_{i} x_{i}^{2}(s)+\sum_{j=1}^{m} \bar{c}_{i} \widetilde{a_{j i}} k_{j}\left(x_{i}^{2}(s)+y_{j}^{2}(s)\right)\right.\right. \\
& \left.+\sum_{j=1}^{m} \bar{c}_{i} \tilde{b}_{j i} k_{j}\left(x_{i}^{2}(s)+y_{j}^{2}(s-\tau(s))\right)+\bar{c}_{i}\left(x_{i}^{2}(s)+I_{i}^{2}(s)\right)\right]+\sum_{j=1}^{m}\left[-2 \underline{d}_{j} y_{j}^{2}(s)+\sum_{i=1}^{n} \bar{d}_{j}{\tilde{c_{i j}}}_{i} l_{i}\left(x_{i}^{2}(s)+y_{j}^{2}(s)\right)\right. \\
& \left.\left.+\sum_{i=1}^{n} \bar{d}_{j}{\widetilde{d_{i j}}}_{i}\left(y_{j}^{2}(s)+x_{i}^{2}(s-\tau(s))\right)+\bar{d}_{j}\left(y_{j}^{2}(s)+J_{j}^{2}(s)\right)\right]\right\} \mathrm{d} s \\
& =V(x(0), y(0), 0)+\int_{0}^{t} \varepsilon V(x(s), y(s), s) \mathrm{d} s+\int_{0}^{t} e^{\varepsilon s}\left\{\sum _ { i = 1 } ^ { n } \left[\left(\bar{c}_{i}-2 \underline{c}_{i}\right) x_{i}^{2}(s)+\sum_{j=1}^{m}\left(\widetilde{a_{j i}}+\widetilde{b_{j i}}\right) \bar{c}_{i} k_{j} x_{i}^{2}(s)\right.\right. \\
& \left.+\sum_{j=1}^{m} \widetilde{c_{i j}} l_{i} \bar{d}_{j} x_{i}^{2}(s)+\sum_{i=1}^{n} \bar{d}_{j} \widetilde{d_{i j}} l_{i} x_{i}^{2}(s-\tau(s))+\bar{c}_{i} I_{i}^{2}(s)\right]+\sum_{j=1}^{m}\left[\left(\bar{d}_{j}-2 \underline{d}_{j}\right) y_{j}^{2}(s)+\sum_{i=1}^{n} \bar{d}_{j}\left(\widetilde{c_{i j}}+\widetilde{d_{i j}}\right) l_{i} y_{j}^{2}(s)\right. \\
& \left.\left.+\sum_{i=1}^{n} \bar{c}_{i} \widetilde{a_{j i}} k_{j} y_{j}^{2}(s)+\sum_{i=1}^{n} \bar{c}_{i} \tilde{b}_{j i} k_{j} y_{j}^{2}(s-\tau(s))+\bar{d}_{j} J_{j}^{2}(s)\right]\right\} \mathrm{d} s \\
& =V(x(0), y(0), 0)+\int_{0}^{t} \varepsilon V(x(s), y(s), s) \mathrm{d} s+\int_{0}^{t} e^{\varepsilon s}\left\{\sum _ { i = 1 } ^ { n } \left[\left[\left(\bar{c}_{i}-2 \underline{c}_{i}\right)+\sum_{j=1}^{m}\left(\widetilde{a_{j i}}+\widetilde{b_{j i}}\right) \bar{c}_{i} k_{j}+\sum_{j=1}^{m} \widetilde{c_{i j}} l_{i} \bar{d}_{j} x_{i}^{2}(s)\right.\right.\right. \\
& +\bar{c}_{i} I_{i}^{2}(s)+\sum_{j=1}^{m} \sum_{i=1}^{n} \bar{d}_{j} \widetilde{d_{i j}} l_{i} x_{i}^{2}(s-\tau(s))+\sum_{j=1}^{m}\left[\left(\bar{d}_{j}-2 \underline{d}_{j}\right)+\sum_{i=1}^{n} \bar{d}_{j}\left(\widetilde{c_{i j}}+\widetilde{d_{i j}}\right) l_{i}\right. \\
& \left.\left.\left.+\sum_{i=1}^{n} \bar{c}_{i} \widetilde{a_{j i}} k_{j}\right] y_{j}^{2}(s)+\sum_{j=1}^{m} \sum_{i=1}^{n} \bar{c}_{i} \tilde{b_{j i}} k_{j} y_{j}^{2}(s-\tau(s))+\bar{d}_{j} J_{j}^{2}(s)\right]\right\} \mathrm{d} s .
\end{aligned}
$$


By exchanging the integrals, we get

$$
\begin{aligned}
& \int_{0}^{t} e^{\varepsilon s} \sum_{i=1}^{n} \sum_{j=1}^{m} \widetilde{d_{i j}} l_{i} x_{i}^{2}(s-\tau(s)) \bar{d}_{j} \mathrm{~d} s \leq \frac{1}{1-\mu} \int_{-\tau}^{0} e^{\varepsilon(s+\tau)} \sum_{i=1}^{n} \sum_{j=1}^{m} \widetilde{d_{i j}} l_{i} \bar{d}_{j} x_{i}^{2}(s) \mathrm{d} s+\frac{1}{1-\mu} \int_{0}^{t} e^{\varepsilon(s+\tau)} \sum_{i=1}^{n} \sum_{j=1}^{m} \widetilde{d_{i j}} l_{i} \bar{d}_{j} x_{i}^{2}(s) \mathrm{d} s \\
& \int_{0}^{t} e^{\varepsilon s} \sum_{i=1}^{n} \sum_{j=1}^{m} \widetilde{b_{j i}} k_{j} \bar{c}_{i} y_{j}^{2}(s-\tau(s)) \mathrm{d} s \leq \frac{1}{1-\mu} \int_{-\tau}^{0} e^{\varepsilon(s+\tau)} \sum_{i=1}^{n} \sum_{j=1}^{m} \widetilde{b_{j i}} k_{j} \bar{c}_{i} y_{j}^{2}(s) \mathrm{d} s+\frac{1}{1-\mu} \int_{0}^{t} e^{\varepsilon(s+\tau)} \sum_{i=1}^{n} \sum_{j=1}^{m} \tilde{b_{j i}} k_{j} \bar{c}_{i} y_{j}^{2}(s) \mathrm{d} s .
\end{aligned}
$$

\section{Combining (17) and (18), then}

$V(x(t), y(t), t)$

$$
\begin{aligned}
& \leq V(x(0), y(0), 0)+\int_{0}^{t} \varepsilon V(x(s), y(s), s) \mathrm{d} s+\frac{e^{\varepsilon \tau}}{1-\mu} \int_{-\tau}^{0} e^{\varepsilon s} \sum_{i=1}^{n} \sum_{j=1}^{n} \widetilde{d_{i j}} \widetilde{l_{i}} \bar{d}_{j} x_{i}^{2}(s) \mathrm{d} s \\
& \quad+\int_{0}^{t} e^{\varepsilon s}\left\{\sum_{i=1}^{n}\left[\bar{c}_{i}-2 \underline{c}_{i}+\sum_{j=1}^{m}\left[\left(\widetilde{a_{j i}}+\widetilde{b_{j i}}\right) k_{j} \bar{c}_{i}+\widetilde{c_{i j}} l_{i} \bar{d}_{j}\right]+\frac{e^{\varepsilon \tau}}{1-\mu} \sum_{j=1}^{m} \widetilde{d_{i j}} l_{i} \bar{d}_{j} x_{i}^{2}(s)\right]+\sum_{i=1}^{n} \bar{c} I_{i}^{2}(s)_{i}\right\} \mathrm{d} s \\
& +\frac{e^{\varepsilon \tau}}{1-\mu} \int_{-\tau}^{0} e^{\varepsilon s} \sum_{i=1}^{n} \sum_{j=1}^{m} \widetilde{b_{j i}} k_{j} \bar{c}_{i} y_{j}^{2}(s) \mathrm{d} s+\int_{0}^{t} e^{\varepsilon s}\left\{\sum_{j=1}^{m}\left[\bar{d}_{j}-2 \underline{d}_{j}+\sum_{i=1}^{n}\left[\left(\widetilde{c_{i j}}+\widetilde{d_{i j}}\right) l_{i} \bar{d}_{j}+\widetilde{a_{j i}} k_{j} \bar{c}_{i}\right] y_{j}^{2}(s)+\frac{e^{\varepsilon \tau}}{1-\mu} \sum_{i=1}^{n} \widetilde{b_{j i}} k_{j} \bar{c}_{i}\right] y_{j}^{2}(s)\right. \\
& \left.+\sum_{j=1}^{m} \bar{d}_{j} J_{j}^{2}(s)\right\} \mathrm{d} s
\end{aligned}
$$$$
\leq V(x(0), y(0), 0)+\int_{0}^{t} \varepsilon e^{\varepsilon s}\left(\sum_{i=1}^{n} x_{i}^{2}(s)+\sum_{j=1}^{m} y_{j}^{2}(s)\right) \mathrm{d} s+\frac{e^{\varepsilon \tau}}{1-\mu} \int_{-\tau}^{0} e^{\varepsilon s} \sum_{i=1}^{n} \sum_{j=1}^{m} \widetilde{d}_{i j} l_{i} \bar{d}_{j} x_{i}^{2}(s) \mathrm{d} s
$$$$
+\int_{0}^{t} e^{\varepsilon s}\left\{\sum_{i=1}^{n}\left[\bar{c}_{i}-2 \underline{c}_{i}+\sum_{j=1}^{m}\left[\left(\widetilde{a_{j i}}+\widetilde{b_{j i}}\right) k_{j} \bar{c}_{i}+\widetilde{c_{i j}} l_{i} \bar{d}_{j}\right]+\frac{e^{\varepsilon \tau}}{1-\mu} \sum_{j=1}^{m} \widetilde{d_{i j}} l_{i} \bar{d}_{j}\right] x_{i}^{2}(s) \mathrm{d} s\right\} \frac{e^{\varepsilon \tau}}{1-\mu} \int_{-\tau}^{0} e^{\varepsilon s} \sum_{i=1}^{n} \sum_{j=1}^{m} \widetilde{b_{j i}} k_{j} \bar{c}_{i} y_{j}^{2}(s) \mathrm{d} s
$$$$
+\int_{0}^{t} e^{\varepsilon s}\left\{\sum_{j=1}^{m}\left[\bar{d}_{j}-2 \underline{d}_{j}+\sum_{i=1}^{n}\left[\left(\widetilde{c_{i j}}+\widetilde{d_{i j}}\right) l_{i} \bar{d}_{j}+\widetilde{a_{j i}} k_{j} \bar{c}_{i}\right] y_{j}^{2}(s)+\frac{e^{\varepsilon \tau}}{1-\mu} \sum_{i=1}^{n} \tilde{b_{j i}} k_{j} \bar{c}_{i}\right] y_{j}^{2}(s)\right\} \mathrm{d} s+\int_{0}^{t} e^{\varepsilon s}\left[\sum_{i=1}^{n} \bar{c}_{i} I_{i}^{2}(s)+\sum_{j=1}^{m} \bar{d}_{j} J_{j}^{2}(s)\right] \mathrm{d} s
$$$$
\leq\left(\sum_{i=1}^{n} x_{i}^{2}(0)+\sum_{j=1}^{m} y_{j}^{2}(0)\right)+\frac{e^{\varepsilon \tau}}{1-\mu} \int_{-\tau}^{0} e^{\varepsilon s} \sum_{i=1}^{n} \sum_{j=1}^{m} \widetilde{d_{i j}} l_{i} \bar{d}_{j} x_{i}^{2}(s) \mathrm{d} s+\int_{0}^{t} e^{\varepsilon s} \sum_{i=1}^{n}\left[\varepsilon+\bar{c}_{i}-2 \underline{c}_{i}+\sum_{j=1}^{m}\left[\left(\widetilde{a_{j i}}+\widetilde{b_{j i}}\right) k_{j} \bar{c}_{i}+\widetilde{c_{i j}} l_{i} \bar{d}_{j}\right]\right.
$$$$
\left.+\frac{e^{\varepsilon \tau}}{1-\mu} \sum_{j=1}^{m} \widetilde{d_{i j}} l_{i} \bar{d}_{j}\right] x_{i}^{2}(s) \mathrm{d} s+\frac{e^{\varepsilon \tau}}{1-\mu} \int_{-\tau}^{0} e^{\varepsilon s} \sum_{i=1}^{n} \sum_{j=1}^{m} \widetilde{b_{j i}} k_{j} \bar{c}_{i} y_{j}^{2}(s) \mathrm{d} s+\int_{0}^{t} e^{\varepsilon s}\left\{\sum _ { j = 1 } ^ { m } \left[\varepsilon+\bar{d}_{j}-2 \underline{d}_{j}+\sum_{i=1}^{n}\left[\left(\widetilde{c_{i j}}+\widetilde{d_{i j}}\right) l_{i} \bar{d}_{j}+\widetilde{a_{j i}} k_{j} \bar{c}_{i}\right] y_{j}^{2}(s)\right.\right.
$$$$
\left.\left.+\frac{e^{\varepsilon \tau}}{1-\mu} \sum_{i=1}^{n} \tilde{b_{j i}} k_{j} \bar{c}_{i}\right] y_{j}^{2}(s)\right\} \mathrm{d} s+\int_{0}^{t} e^{\varepsilon s}\left[\sum_{i=1}^{n} \bar{c}_{i} I_{i}^{2}(s)+\sum_{j=1}^{m} \bar{d}_{i} J_{j}^{2}(s)\right] \mathrm{d} s
$$$$
\leq\left(\sum_{i=1}^{n} x_{i}^{2}(0)+\sum_{m=1}^{m} y_{j}^{2}(0)\right)+\frac{1}{1-\mu} \int_{-\tau}^{0} e^{\varepsilon(s+\tau)} \sum_{i=1}^{n} \sum_{j=1}^{m} d_{i j} l_{i} \widetilde{d_{j} x_{i}^{2}}(s) \mathrm{d} s+\frac{1}{1-\mu} \int_{-\tau}^{0} e^{\varepsilon(s+\tau)} \sum_{i=1}^{n} \sum_{j=1}^{m} b_{j i} k_{j} \widetilde{\bar{c}_{i} y_{j}^{2}}(s) \mathrm{d} s+\frac{\bar{c} n}{\varepsilon}\|I\|_{\text {sup }}^{2} e^{\varepsilon t}+\frac{\bar{d} m}{\varepsilon}\|J\|_{\text {sup }}^{2} e^{\varepsilon t}
$$

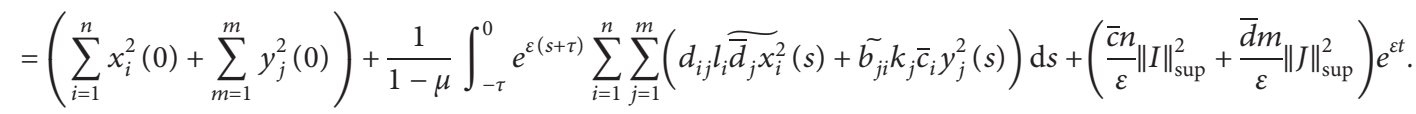


Thus, it follows that

$$
\begin{aligned}
\sum_{i=1}^{n} x_{i}^{2}(t)+\sum_{m=1}^{m} y_{j}^{2}(t) \leq & e^{-\varepsilon t}\left\{\sum_{i=1}^{n} x_{i}^{2}(0)+\sum_{j=1}^{m} y_{j}^{2}(0)+\frac{1}{1-\mu} \int_{-\tau}^{0} e^{\varepsilon(s+\tau)} \sum_{i=1}^{n} \sum_{j=1}^{m}\left({\widetilde{d_{i j}}}_{i j} \bar{d}_{j} x_{i}^{2}(s)+\tilde{b}_{j i} k_{j} \bar{c}_{j} y_{j}^{2}(s)\right) \mathrm{d} s\right. \\
& \left.+\left(\frac{\bar{c} n}{\varepsilon}\|I\|_{\text {sup }}^{2}+\frac{\bar{d} m}{\varepsilon}\|J\|_{\text {sup }}^{2}\right) e^{\varepsilon t}\right\} .
\end{aligned}
$$

Furthermore, $\quad\left(\|x\|_{\infty}+\|y\|_{\infty}\right)^{2} \leq 2\left(\sum_{i=1}^{n} x_{i}^{2}(t)+\sum_{j=1}^{m} y_{j}^{2}\right.$

$(t))$; thus

$$
\begin{aligned}
\|x\|_{\infty}+\|y\|_{\infty} \leq & \sqrt{2} e^{-(\varepsilon / 2) t}\left[\left(\sum_{i=1}^{n} x_{i}^{2}(0)+\sum_{j=1}^{m} y_{j}^{2}(0)+\frac{1}{1-\mu} \int_{-\tau}^{0} e^{\varepsilon(s+\tau)} \sum_{i=1}^{n} \sum_{j=1}^{m}\left(\widetilde{d_{i j}} l_{i} \bar{d}_{j} x_{i}^{2}(s)+\widetilde{b_{j i}} k_{j} \bar{c}_{i} y_{j}^{2}(s)\right) \mathrm{d} s\right]^{1 / 2}\right. \\
& +\left(\sqrt{\left(\frac{2 \bar{c} n}{\varepsilon}\right.}\|I\|_{\text {sup }}+\sqrt{\frac{2 \bar{d} m}{\varepsilon}}\|J\|_{\text {sup }}\right)
\end{aligned}
$$

and from the definition, system (1) is input-to-state stable.

Now, we consider some special cases. For $\tau(t)=0$, system (1) becomes

$$
\left\{\begin{array}{l}
\dot{x}_{i}(t)=c_{i}\left(x_{i}(t)\right)\left[-x_{i}(t)+\sum_{j=1}^{m} a_{j i}\left(x_{i}(t)\right) f_{j}\left(y_{j}(t)\right)+I_{i}(t)\right], \\
\dot{y}_{j}(t)=d_{i}\left(y_{j}(t)\right)\left[-y_{j}(t)+\sum_{i=1}^{n} c_{i j}\left(y_{j}(t)\right) g_{i}\left(x_{i}(t)\right)+J_{j}(t)\right], \\
t \geq 0, i=1,2, \ldots, n ; j=1,2, \ldots, m .
\end{array}\right.
$$

Corollary 1. Assume that (H1)-(H3) hold. Then, system (22) is input-to-state stable if

$$
\begin{aligned}
& \bar{c}_{i}-2 \underline{c}_{i}+\sum_{j=1}^{m} \widetilde{a_{j i}} \bar{c}_{i} k_{j}+\sum_{j=1}^{m} \widetilde{c_{i j}} \bar{d}_{j} l_{i}<0, \quad i=1,2, \ldots, n, \\
& \bar{d}_{j}-2 \underline{d}_{j}+\sum_{i=1}^{n} \widetilde{c_{i j}} \bar{d}_{j} l_{i}+\sum_{i=1}^{n} \widetilde{a_{j i}} \bar{c}_{i} k_{j}<0, \quad j=1,2, \ldots, m . \\
& \text { When } \tau(t)=\tau, \text { system (1) becomes }
\end{aligned}
$$

Corollary 2. Assume that (H1)-(H3) hold. Then, system (24) is input-to-state stable if

$$
\begin{gathered}
\bar{c}_{i}-2 \underline{c}_{i}+\sum_{j=1}^{m}\left(\widetilde{a_{j i}}+\widetilde{b_{j i}}\right) \bar{c}_{i} k_{j}+\sum_{j=1}^{m}\left(\widetilde{c_{i j}}+\widetilde{d_{i j}}\right) \bar{d}_{j} l_{i}<0, \quad i=1,2, \ldots, n, \\
\bar{d}_{j}-2 \underline{d}_{j}+\sum_{i=1}^{n}\left(\widetilde{c_{i j}}+\widetilde{d_{i j}}\right) \bar{d}_{j} l_{i}+\sum_{i=1}^{n}\left(\widetilde{a_{j i}}+\widetilde{b_{j i}}\right) \bar{c}_{i} k_{j}<0, \quad j=1,2, \ldots, m .
\end{gathered}
$$


Remark 2. It is worth noting that the result of Theorem 1 can be improved and simplified. Next, we give related results, which are less conservative than Theorem 1 and are more useful.

Theorem 2. Assume that (H1)-(H3) hold. Then, system (1) is input-to-state stable if

$$
\begin{aligned}
& -\underline{c}_{i}+\sum_{j=1}^{m} \bar{d}_{j}\left(\tilde{c}_{i j}+\frac{\tilde{d}_{i j}}{1-\mu}\right) l_{i}<0, \quad i=1,2, \ldots, n, \\
& -\underline{d}_{j}+\sum_{i=1}^{n} \bar{c}_{i}\left(\tilde{a}_{j i}+\frac{\tilde{b}_{j i}}{1-\mu}\right) k_{j}<0, \quad j=1,2, \ldots, m .
\end{aligned}
$$

Proof. Consider Lyapunov function

$$
\begin{aligned}
V(x(t), y(t), t)= & \left(\sum_{i=1}^{n}\left|x_{i}(t)\right|+\sum_{j=1}^{m}\left|y_{j}(t)\right|\right) \\
& +\frac{1}{1-\mu} \sum_{i=1}^{n} \sum_{j=1}^{m} \int_{t-\tau(t)}^{t}\left(\bar{c}_{i} \widetilde{b}_{j i}\left|f_{j}\left(y_{j}(s)\right)\right|\right) \\
& +\bar{d}_{j} \widetilde{d_{i j}}\left|g_{i}\left(x_{i}(s)\right)\right| \mathrm{d} s .
\end{aligned}
$$

Then, the time derivative of $\mathrm{V}$ along the trajectory of (9) or (10) gives

$$
\begin{aligned}
& D^{+} V(x(t), y(t), t) \\
& \leq \sum_{i=1}^{n}\left[-\underline{c}_{i}\left|x_{i}(t)\right|+\sum_{j=1}^{m} \bar{c}_{i} \tilde{a}_{j i}\left|f_{j}\left(y_{j}(t)\right)\right|+\sum_{j=1}^{n} \bar{c}_{i} \tilde{b}_{j i}\left|f_{j}\left(y_{j}(t-\tau(t))\right)\right|+\bar{c}_{i}\left|I_{i}(t)\right|\right] \\
& +\frac{1}{1-\mu} \sum_{i=1}^{n} \sum_{j=1}^{m} \bar{c}_{i} \tilde{b}_{j i}\left[\left|f_{j}\left(y_{j}(t)\right)\right|-\left|f_{j}\left(y_{j}(t-\tau(t))\right)\right|(1-\mu)\right]+\sum_{j=1}^{m}\left[-\underline{d}_{j}\left|y_{j}(t)\right|+\sum_{i=1}^{n} \bar{d}_{j} \tilde{c}_{i j}\left|g_{i}\left(x_{i}(t)\right)\right|\right. \\
& \left.+\sum_{i=1}^{n} \bar{d}_{j} \widetilde{d}_{i j}\left|g_{i}\left(x_{i}(t-\tau(t))\right)\right|+\bar{d}_{j}\left|J_{j}(t)\right|\right]+\frac{1}{1-\mu} \sum_{i=1}^{n} \sum_{j=1}^{m} \bar{d}_{j} \widetilde{d}_{i j}\left[\left|g_{i}\left(x_{i}(t)\right)\right|-\left|g_{i}\left(x_{i}(t-\tau(t))\right)\right|(1-\mu)\right] \\
& =\sum_{i=1}^{n}\left[-\underline{c}_{i}\left|x_{i}(t)\right|+\sum_{j=1}^{m} \bar{c}_{i} \tilde{a}_{j i}\left|f_{j}\left(y_{j}(t)\right)\right|+\bar{c}_{i}\left|I_{i}(t)\right|\right]+\frac{1}{1-\mu} \sum_{i=1}^{n} \sum_{j=1}^{m} \bar{c}_{i} \tilde{b}_{j i}\left|f_{j}\left(y_{j}(t)\right)\right|+\sum_{j=1}^{m}\left[-\underline{d}_{j}\left|y_{j}(t)\right|+\sum_{n=1}^{n} \bar{d}_{j} \tilde{c}_{i j}\left|g_{i}\left(x_{i}(t)\right)\right|\right. \\
& \left.+\bar{d}_{j}\left|J_{j}(t)\right|\right]+\frac{1}{1-\mu} \sum_{i=1}^{n} \sum_{j=1}^{m} \bar{d}_{j} \widetilde{d}_{i j}\left|g_{i}\left(x_{i}(t)\right)\right| \\
& \left.\leq-\sum_{i=1}^{n}\left|\underline{c}_{i} x_{i}(t)\right|+\sum_{i=1}^{n} \sum_{j=1}^{m} \bar{c}_{i}\left(\tilde{a}_{j i}+\frac{1}{1-\mu} \tilde{b_{j i}}\right) k_{j}\left|y_{j}(t)\right|\right)+\sum_{i=1}^{n} \bar{c}_{i}\left|I_{i}(t)\right|-\sum_{j=1}^{m} \underline{d}_{j}\left|y_{j}(t)\right|+\sum_{j=1}^{m} \sum_{i=1}^{n} \bar{d}_{j}\left(\tilde{c_{i j}}+\frac{1}{1-\mu} \tilde{d}_{i j}\right) l_{i}\left|x_{i}(t)\right| \\
& +\sum_{j=1}^{m} \bar{d}_{j}\left|J_{j}(t)\right| \\
& \leq-\sum_{i=1}^{n}\left[\underline{c}_{i}-\sum_{j=1}^{m} \bar{d}_{j}\left(\tilde{c_{i j}}+\frac{1}{1-\mu} \tilde{d}_{i j}\right) l_{i}\right]\left|x_{i}(t)\right|+\sum_{i=1}^{n} \bar{c}_{i}\left|I_{i}(t)\right|-\sum_{j=1}^{m}\left[\underline{d}_{j}-\sum_{i=1}^{n} \bar{c}_{i}\left(\tilde{a}_{j i}+\frac{1}{1-\mu} \tilde{b_{j i}}\right) k_{j}\right]\left|y_{j}(t)\right|+\sum_{j=1}^{m} \bar{d}_{j}\left|J_{j}(t)\right| \\
& \leq-\delta\left(\sum_{i=1}^{n}\left|x_{i}(t)\right|+\sum_{j=1}^{m}\left|y_{j}(t)\right|\right)+\left(\sum_{i=1}^{n} \bar{c}_{i}\left|I_{i}(t)\right|+\sum_{j=1}^{m} \bar{d}_{j}\left|J_{j}(t)\right|\right)
\end{aligned}
$$

where $\delta=\min \{\eta, \nu\}, \eta=\min _{1 \leq i \leq n}\left(\underline{c}_{i}-\sum_{j=1}^{m} \bar{d}_{j}\left(\widetilde{c}_{i j}+(1 /(1-\right.\right.$ $\left.\left.\mu)) \widetilde{d}_{i j}\right) l_{i}\right)>0, v=\min _{1 \leq j \leq m}\left(\underline{d}_{j}-\sum_{i=1}^{n} \bar{c}_{i}\left(\widetilde{a}_{j i}+(1 /(1-\mu)) \widetilde{b}_{j i}\right)\right.$ $\left.k_{j}\right)>0$. For any $1 \leq i \leq n$, choose $\varepsilon_{i}>0$ and any $1 \leq j \leq m$, choose $\varepsilon_{j}>0$, respectively, and we correspondingly have 
Complexity

9

$$
\begin{aligned}
& -\delta+\varepsilon_{i}+\frac{1}{1-\mu} \sum_{j=1}^{m} \bar{d}_{j} \widetilde{d}_{i j} l_{i} \varepsilon_{i} \tau e^{\varepsilon_{i} \tau}=0, \\
& -\delta+\varepsilon_{j}+\frac{1}{1-\mu} \sum_{j=1}^{n} \bar{c}_{i} \widetilde{b}_{j i} k_{j} \varepsilon_{j} \tau e^{\varepsilon_{j} \tau}=0 .
\end{aligned}
$$

Let $\varepsilon=\min \left\{\min _{1 \leq i \leq n}\left(\varepsilon_{i}\right), \min _{1 \leq j \leq n}\left(\varepsilon_{j}\right)\right\}>0$.

For any $1 \leq i \leq n$ and any $1 \leq j \leq m$, respectively, we correspondingly obtained

$$
\begin{aligned}
& -\delta+\varepsilon+\frac{\varepsilon \tau e^{\varepsilon \tau}}{1-\mu} \sum_{j=1}^{m} \bar{d}_{j} \widetilde{d}_{i j} l_{i} \leq 0, \\
& -\delta+\varepsilon+\frac{\varepsilon \tau e^{\varepsilon \tau}}{1-\mu} \sum_{i=1}^{n} \bar{c}_{i} \widetilde{b}_{j i} k_{j} \leq 0 .
\end{aligned}
$$

Let $W(x(t), y(t), t)=e^{\varepsilon t} V(x(t), y(t), t)$. Calculating the time derivative of $W$ along the trajectory of (9) or (10), we yield

$$
\begin{aligned}
& D^{+} W(x(t), y(t), t) \\
& =\varepsilon e^{\varepsilon t} V(x(t), y(t), t)+e^{\varepsilon t} D^{+} V(x(t), y(t), t) \\
& \leq \varepsilon e^{\varepsilon t}\left[\sum_{i=1}^{n}\left|x_{i}(t)\right|+\sum_{i=1}^{m}\left|y_{j}(t)\right|+\frac{1}{1-\mu} \sum_{i=1}^{n} \sum_{j=1}^{m} \int_{t-\tau(t)}^{t} \bar{c}_{i} \widetilde{b_{j i}}\left|f_{j}\left(y_{j}(s)\right)\right|+\bar{d}_{j} \widetilde{d_{i j}}\left|g_{i}\left(x_{i}(s)\right)\right|\right] \mathrm{d} s-\delta e^{\varepsilon t}\left(\sum_{i=1}^{n}\left|x_{i}(t)\right|+\sum_{j=1}^{m}\left|y_{j}(t)\right|\right) \\
& \quad+e^{\varepsilon t}\left(\sum_{i=1}^{n} \bar{c}_{i}\left|I_{i}(t)\right|+\sum_{j=1}^{m} \bar{d}_{j}\left|J_{j}(t)\right|\right) \\
& \leq(-\delta+\varepsilon) e^{\varepsilon t}\left(\sum_{i=1}^{n}\left|x_{i}(t)\right|+\sum_{j=1}^{m}\left|y_{j}(t)\right|\right)+\frac{1}{1-\mu} \sum_{i=1}^{n} \sum_{j=1}^{m} \varepsilon e^{\varepsilon t} \int_{t-\tau(t)}^{t}\left(\bar{c}_{i} k_{j} \widetilde{b_{j i}}\left|y_{j}(s)\right|+\bar{d}_{j} l_{i} \widetilde{d_{i j}}\left|x_{i}(s)\right|\right) \mathrm{d} s \\
& \quad+e^{\varepsilon t}\left(\sum_{i=1}^{n} \bar{c}_{i}\left|I_{i}(t)\right|+\sum_{j=1}^{m} \bar{d}_{j}\left|J_{j}(t)\right|\right) .
\end{aligned}
$$

Integrating both sides of (31), we have

$$
\begin{aligned}
& W(x(t), y(t), t) \\
& \leq W\left(x_{0}, y_{0}, 0\right)+\sum_{i=1}^{n}(-\delta+\varepsilon) \int_{0}^{t} e^{\varepsilon s}\left|x_{i}(s)\right| \mathrm{d} s+\sum_{j=1}^{m}(-\delta+\varepsilon) \int_{0}^{t} e^{\varepsilon s}\left|y_{j}(s)\right| \mathrm{d} s \\
& \quad+\frac{\varepsilon}{1-\mu} \sum_{i=1}^{n} \sum_{j=1}^{m}\left(\bar{c}_{i} \widetilde{b_{j i}} k_{j} \int_{0}^{t} e^{\varepsilon s} \int_{s-\tau}^{s}\left|y_{j}(v)\right| \mathrm{d} v \mathrm{~d} s+\bar{d}_{j} \widetilde{d_{i j}} l_{i} \int_{0}^{t} e^{\varepsilon s} \int_{s-\tau}^{s}\left|x_{i}(v)\right| \mathrm{d} v \mathrm{~d} s\right)+\sum_{i=1}^{n} \int_{0}^{t} e^{\varepsilon s} \bar{c}_{i}\left|I_{i}(s)\right| \mathrm{d} s++\sum_{j=1}^{m} \int_{0}^{t} e^{\varepsilon s} \bar{d} j\left|J_{j}(s)\right| \mathrm{d} s \\
& \leq V\left(x_{0}, y_{0}, 0\right)+\sum_{i=1}^{n}(-\delta+\varepsilon) \int_{0}^{t} e^{\varepsilon s}\left|x_{i}(s)\right| \mathrm{d} s+\sum_{j=1}^{m}(-\delta+\varepsilon) \int_{0}^{t} e^{\varepsilon s}\left|y_{j}(s)\right| \mathrm{d} s \\
& \quad+\frac{\varepsilon}{1-\mu} \sum_{i=1}^{n} \sum_{j=1}^{m}\left(\bar{c}_{i} \tilde{b_{j i}} k_{j} \int_{0}^{t} e^{\varepsilon s} \int_{s-\tau}^{s}\left|y_{j}(v)\right| \mathrm{d} v \mathrm{~d} s+\bar{d}_{j} \widetilde{d_{i j}} l_{i} \int_{0}^{t} e^{\varepsilon s} \int_{s-\tau}^{s}\left|x_{i}(v)\right| \mathrm{d} v \mathrm{~d} s\right)+\left(\frac{\bar{c} n_{n}}{\varepsilon}\|I\|_{\text {sup }}+\frac{\bar{d} m}{\varepsilon}\|J\|_{\text {sup }}\right) e^{\varepsilon t} .
\end{aligned}
$$

While 
10

Complexity

$$
\begin{aligned}
& \frac{\varepsilon}{1-\mu} \sum_{i=1}^{n} \sum_{j=1}^{m} \tilde{b_{j i}} k_{j} \int_{0}^{t} e^{\varepsilon s} \int_{s-\tau}^{s}\left|y_{j}(v)\right| \mathrm{d} v \mathrm{~d} s \\
& =\frac{\varepsilon}{1-\mu} \sum_{i=1}^{n} \sum_{j=1}^{m} \tilde{b_{j i}} k_{j} \int_{-\tau}^{t}\left|y_{j}(v)\right| \int_{\max (0, v)}^{\min (t, v+t)} e^{\varepsilon s} \mathrm{~d} v \mathrm{~d} s \\
& \leq \frac{\varepsilon \tau e^{\varepsilon \tau}}{1-\mu} \sum_{i=1}^{n} \sum_{j=1}^{m} \tilde{b_{j i}} k_{j} \int_{-\tau}^{t}\left|e^{\varepsilon v} y_{j}(v)\right| \mathrm{d} v \\
& =\frac{\varepsilon \tau e^{\varepsilon \tau}}{1-\mu} \sum_{i=1}^{n} \sum_{j=1}^{m} \tilde{b_{j i}} k_{j} \int_{-\tau}^{0} e^{\varepsilon s}\left|y_{j}(s)\right| \mathrm{d} s+\frac{\varepsilon \tau e^{\varepsilon \tau}}{1-\mu} \sum_{i=1}^{n} \sum_{j=1}^{m} \tilde{b_{j i}} k_{j} \int_{0}^{t} e^{\varepsilon s}\left|y_{j}(s)\right| \mathrm{d} s, \\
& \frac{\varepsilon}{1-\mu} \sum_{i=1}^{n} \sum_{j=1}^{m} \widetilde{d_{i j}} l_{i} \int_{0}^{t} e^{\varepsilon s} \int_{s-\tau}^{s}\left|x_{i}(v)\right| \mathrm{d} v \mathrm{~d} s \\
& =\frac{\varepsilon}{1-\mu} \sum_{i=1}^{n} \sum_{j=1}^{m} \widetilde{d_{i j}} l_{i} \int_{-\tau}^{t}\left|x_{i}(v)\right| \int_{\max (0, v)}^{\min (t, v+t)} e^{\varepsilon s} \mathrm{~d} v \mathrm{~d} s \\
& \leq \frac{\varepsilon \tau e^{\varepsilon \tau}}{1-\mu} \sum_{i=1}^{n} \sum_{j=1}^{m} \widetilde{d_{i j}} l_{i} \int_{-\tau}^{t}\left|e^{\varepsilon v} x_{i}(v)\right| \mathrm{d} v \\
& =\frac{\varepsilon \tau e^{\varepsilon \tau}}{1-\mu} \sum_{i=1}^{n} \sum_{j=1}^{m} \widetilde{d_{i j}} l_{i} \int_{-\tau}^{0} e^{\varepsilon s}\left|x_{i}(s)\right| \mathrm{d} s+\frac{\varepsilon \tau \tau e^{\varepsilon \tau}}{1-\mu} \sum_{i=1}^{n} \sum_{j=1}^{m} \widetilde{d_{i j}} l_{i} \int_{0}^{t} e^{\varepsilon s}\left|x_{i}(s)\right| \mathrm{d} s .
\end{aligned}
$$

Combining (32) and (33a) and (33b) leads to

$$
\begin{aligned}
& W(x(t), y(t), t) \\
& \leq V\left(x_{0}, y_{0}, 0\right)+\frac{\varepsilon \tau e^{\varepsilon \tau}}{1-\mu} \sum_{i=1}^{n} \sum_{j=1}^{m} \bar{d}_{j} \widetilde{d_{i j}} l_{i} \int_{-\tau}^{0} e^{\varepsilon s}\left|x_{i}(s)\right| \mathrm{d} s+\frac{\varepsilon \tau e^{\varepsilon \tau}}{1-\mu} \sum_{i=1}^{n} \sum_{j=1}^{m} \bar{c}_{i} \widetilde{b_{j i}} k_{j} \int_{-\tau}^{0} e^{\varepsilon s}\left|y_{j}(s)\right| \mathrm{d} s \\
& +\sum_{j=1}^{m}\left(-\delta+\varepsilon+\frac{\varepsilon \tau e^{\varepsilon \tau}}{1-\mu} \sum_{i=1}^{n} \bar{c}_{i} \tilde{b_{j i}} k_{j}\right) \int_{0}^{t} e^{\varepsilon s}\left|y_{j}(s)\right| \mathrm{d} s \\
& +\sum_{i=1}^{n}\left(-\delta+\varepsilon+\frac{\varepsilon \mathcal{E} \tau e^{\varepsilon \tau}}{1-\mu} \sum_{j=1}^{m} \bar{d}_{j} \widetilde{d}_{i j} l_{i}\right) \int_{0}^{t} e^{\varepsilon s}\left|x_{i}(s)\right| \mathrm{d} s+\left(\frac{\bar{c} n}{\varepsilon}\|I\|_{\text {sup }}+\frac{\bar{d} m}{\varepsilon}|J|_{\text {sup }}\right) e^{\varepsilon t} \\
& \leq V\left(x_{0}, y_{0}, 0\right)+\frac{\varepsilon \tau e^{\varepsilon \tau}}{1-\mu} \sum_{i=1}^{n} \sum_{j=1}^{m} \bar{d}_{j} \widetilde{d_{i j}} l_{i} \int_{-\tau}^{0} e^{\varepsilon s}\left|x_{i}(s)\right| \mathrm{d} s+\frac{\varepsilon \tau e^{\varepsilon \tau}}{1-\mu} \sum_{i=1}^{n} \sum_{j=1}^{m} \bar{c}_{i} \widetilde{b}_{j i} k_{j} \int_{-\tau}^{0} e^{\varepsilon s}\left|y_{j}(s)\right| \mathrm{d} s \\
& +\left(\frac{\bar{c} n}{\varepsilon}\|I\|_{\text {sup }}+\frac{\bar{d} m}{\varepsilon}\|J\|_{\text {sup }}\right) e^{\varepsilon t}
\end{aligned}
$$

Thus, it follows that 


$$
\begin{aligned}
V(x(t), y(t), t)= & W(x(t), y(t), t) e^{-\varepsilon t} \\
\leq & {\left[V\left(x_{0}, y_{0}, 0\right)+\frac{\varepsilon \tau e^{\varepsilon \tau}}{1-\mu} \sum_{i=1}^{n} \sum_{j=1}^{m} \overline{d_{j}} \widetilde{d_{i j}} l_{i} \int_{-\tau}^{0} e^{\varepsilon s}\left|x_{i}(s)\right| \mathrm{d} s+\frac{\varepsilon \tau e^{\varepsilon \tau}}{1-\mu} \sum_{i=1}^{n} \sum_{j=1}^{m} \bar{c}_{i} \widetilde{b_{j i}} k_{j} \int_{-\tau}^{0} e^{\varepsilon s}\left|y_{j}(s)\right| \mathrm{d} s\right] e^{-\varepsilon t} } \\
& +\left(\frac{\bar{c} n_{n}}{\varepsilon}\|I\|_{\text {sup }}+\frac{\bar{d} m}{\varepsilon}|J|_{\text {sup }}\right) \\
\leq & {\left[\sum_{i=1}^{n}\left|x_{i}(0)\right|+\sum_{j=1}^{n}\left|y_{j}(0)\right|+\frac{1}{1-\mu} \sum_{i=1}^{n} \sum_{j=1}^{m} \int_{-\tau}^{0} \overline{c_{i}}\left(\widetilde{b_{j i}} k_{j}\left|y_{j}(s)\right|+\bar{d}_{j} \widetilde{d_{i j}} l_{i}\left|x_{i}(s)\right|\right) \mathrm{d} s\right.} \\
& \left.+\frac{\varepsilon \tau e^{\varepsilon \tau}}{1-\mu} \sum_{i=1}^{n} \sum_{j=1}^{m} \bar{d}_{j} \widetilde{d_{i j}} l_{i} \int_{-\tau}^{0} e^{\varepsilon s}\left|x_{i}(s)\right| \mathrm{d} s+\frac{\varepsilon \tau e^{\varepsilon \tau}}{1-\mu} \sum_{i=1}^{n} \sum_{j=1}^{m} \bar{c}_{i} \widetilde{b_{j i}} k_{j} \int_{-\tau}^{0} e^{\varepsilon s}\left|y_{j}(s)\right| \mathrm{d} s\right] e^{-\varepsilon t}+\left(\frac{\bar{c} n_{n}}{\varepsilon}\|I\|_{\text {sup }}+\frac{\bar{d} m}{\varepsilon}\|J\|_{\text {sup }}\right) .
\end{aligned}
$$

Furthermore, $\|x\|_{\infty}+\|y\|_{\infty} \leq\left(\sum_{i=1}^{n}\left|x_{i}(t)\right|+\sum_{j=1}^{m}\left|y_{i}(t)\right|\right)$

$\leq V(x(t), y(t), t)$ such that

$$
\begin{aligned}
\|x\|_{\infty}+\|y\|_{\infty} \leq & {\left[\sum_{i=1}^{n}\left|x_{i}(0)\right|+\sum_{j=1}^{m}\left|y_{j}(0)\right|+\frac{1}{1-\mu} \sum_{i=1}^{n} \sum_{j=1}^{m} \int_{-\tau}^{0}\left(\bar{c}_{i} \widetilde{b_{j i}} k_{j}\left|y_{j}(s)\right|+\bar{d}_{j} \widetilde{d_{i j}} l_{i}\left|x_{i}(s)\right|\right) \mathrm{d} s\right.} \\
& \left.+\frac{\varepsilon \tau e^{\varepsilon \tau}}{1-\mu} \sum_{i=1}^{n} \sum_{j=1}^{m} \bar{d}_{j} \widetilde{d_{i j}} l_{i} \int_{-\tau}^{0} e^{\varepsilon s}\left|x_{i}(s)\right| \mathrm{d} s+\frac{\varepsilon \tau e^{\varepsilon \tau}}{1-\mu} \sum_{i=1}^{n} \sum_{j=1}^{m} \bar{c}_{i} \widetilde{b_{j i}} k_{j} \int_{-\tau}^{0} e^{\varepsilon s}\left|y_{j}(s)\right| \mathrm{d} s\right] e^{-\varepsilon t}+\left(\frac{\bar{c} n}{\varepsilon}\|I\|_{\text {sup }}+\frac{\bar{d} m}{\varepsilon}\|J\|_{\text {sup }}\right),
\end{aligned}
$$

and from the definition, system (1) is input-to-state stable.

Similar to Theorem 1, we have related corollaries for special cases as follows.

Corollary 3. Assume that (H1)-(H3) hold. Then, system (22) is input-to-state stable if

$$
\begin{aligned}
-\underline{c}_{i}+\sum_{j=1}^{m} \bar{d}_{j} \widetilde{c}_{i j} l_{i}<0, \quad i=1,2, \ldots, n ; \\
-\underline{d}_{j}+\sum_{i=1}^{n} \bar{c}_{i} \widetilde{a}_{j i} k_{j}<0, \quad i=1,2, \ldots, m .
\end{aligned}
$$

Corollary 4. Assume that (H1)-(H3) hold. Then, system (24) is input-to-state stable if

$$
\begin{aligned}
& -\underline{c}_{i}+\sum_{j=1}^{m} \bar{d}_{j}\left(\widetilde{c}_{i j}+\widetilde{d}_{i j}\right) l_{i}<0, \quad i=1,2, \ldots, n ; \\
& -\underline{d}_{j}+\sum_{i=1}^{n} \bar{c}_{i}\left(\widetilde{a}_{j i}+\widetilde{b}_{j i}\right) k_{j}<0, \quad i=1,2, \ldots, m .
\end{aligned}
$$

\section{A Numerical Example}

Next, we give an illustrative example to show the effectiveness of our results.

We consider the memristive Cohen-Grossberg BAM neural networks with variable delays as follows:

$$
\left\{\begin{array}{l}
\dot{x}_{1}(t)=c_{1}\left(x_{1}(t)\right)\left[-x_{1}(t)+a_{11}\left(x_{1}(t)\right) f_{2}\left(x_{2}(t)\right)+a_{12}\left(x_{1}(t)\right) f_{2}\left(x_{2}(t-\tau(t))\right)+I_{1}(t)\right], \\
\dot{x}_{2}(t)=c_{2}\left(x_{2}(t)\right)\left[-x_{2}(t)+b_{21}\left(x_{2}(t)\right) f_{1}\left(x_{1}(t)\right)+b_{22}\left(x_{2}(t)\right) f_{1}\left(x_{1}(t-\tau(t))\right)+I_{2}(t)\right],
\end{array}\right.
$$

for $\quad t \geq 0, \quad$ where $\quad c_{1}\left(x_{1}(t)\right)=1+(1 / 16) \cos x_{1}(t)$, $c_{2}\left(x_{2}(t)\right)=1+(1 / 8) \sin x_{2}(t), \tau(t)=e^{-3 t}, I_{1}(t)=0.5 \sin t$, and $I_{2}(t)=0.6 \cos t . f_{1}(x)=f_{2}(x)=\tanh x$ is globally Lipschitzian. Let 


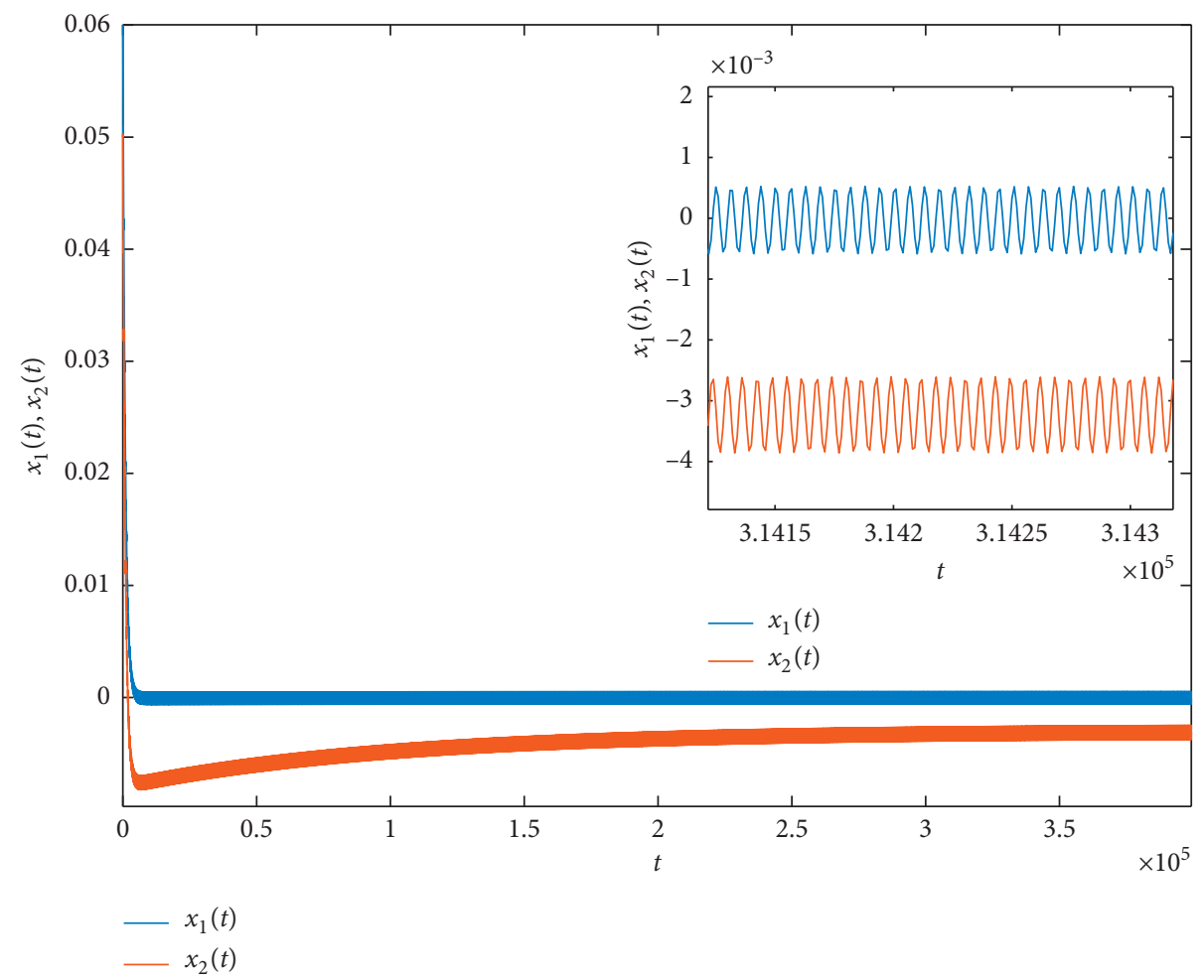

Figure 1: Time evolutions $x_{1}(t), x_{2}(t)$ of system (39).

$$
\begin{aligned}
& a_{11}\left(x_{1}(t)\right)= \begin{cases}0.01, & \frac{\mathrm{d} f_{2}\left(x_{2}(t)\right)}{\mathrm{d} t}-\frac{\mathrm{d} x_{1}(t)}{\mathrm{d} t} \leq 0, \\
-0.01, & \frac{\mathrm{d} f_{2}\left(x_{2}(t)\right)}{\mathrm{d} t}-\frac{\mathrm{d} x_{1}(t)}{\mathrm{d} t}>0,\end{cases} \\
& a_{12}\left(x_{1}(t)\right)= \begin{cases}0.02, & \frac{\mathrm{d} f_{2}\left(x_{2}(t-\tau(t))\right)}{\mathrm{d} t}-\frac{\mathrm{d} x_{1}(t)}{\mathrm{d} t} \leq 0, \\
-0.02, & \frac{d f_{2}\left(x_{2}(t-\tau(t))\right)}{\mathrm{d} t}-\frac{\mathrm{d} x_{1}(t)}{\mathrm{d} t}>0,\end{cases} \\
& b_{21}\left(x_{2}(t)\right)= \begin{cases}0.02, & \frac{\mathrm{d} f_{1}\left(x_{1}(t)\right)}{\mathrm{d} t}-\frac{\mathrm{d} x_{2}(t)}{\mathrm{d} t} \leq 0, \\
-0.02, & \frac{\mathrm{d} f_{1}\left(x_{1}(t)\right)}{\mathrm{d} t}-\frac{\mathrm{d} x_{2}(t)}{\mathrm{d} t}>0,\end{cases} \\
& b_{22}\left(x_{2}(t)\right)= \begin{cases}0.03, & \frac{\mathrm{d} f_{1}\left(x_{1}(t-\tau(t))\right)}{\mathrm{d} t}-\frac{\mathrm{d} x_{2}(t)}{\mathrm{d} t} \leq 0, \\
-0.03, & \frac{d f_{1}\left(x_{1}(t-\tau(t))\right)}{\mathrm{d} t}-\frac{\mathrm{d} x_{2}(t)}{\mathrm{d} t}>0 .\end{cases}
\end{aligned}
$$

From Theorem 1, we get

$$
\begin{array}{r}
\frac{17}{16}-\frac{15}{16} \times 2+(0.01+0.02) \times \frac{17}{16}+(0.02+0.03) \times \frac{17}{16} \approx-0.68<0, \\
\frac{9}{8}-\frac{7}{8} \times 2+(0.01+0.02) \times \frac{9}{8}+(0.02+0.03) \times \frac{9}{8} \approx-0.54<0 .
\end{array}
$$

From Theorem 2, we obtain

$$
\begin{aligned}
& -\frac{15}{16}+(0.02+0.03) \times \frac{9}{8} \approx-0.88<0, \\
& -\frac{7}{8}+(0.01+0.02) \times \frac{17}{16} \approx-0.84<0 .
\end{aligned}
$$

Based on the calculation, it is easy to know that the conditions of Theorem 1 and Theorem 2 are satisfied. Thus, system (39) is input-to-state stable. When initial values $x(0)=[0.06 ; 0.05]^{T}$, Figure 1 shows that time evolutions of system (39) with periodic inputs are bounded. Therefore, it is input-to-state stable. When inputs are zeros, Figure 2 shows that the state of system (39) with identical initial values is exponentially stable. 


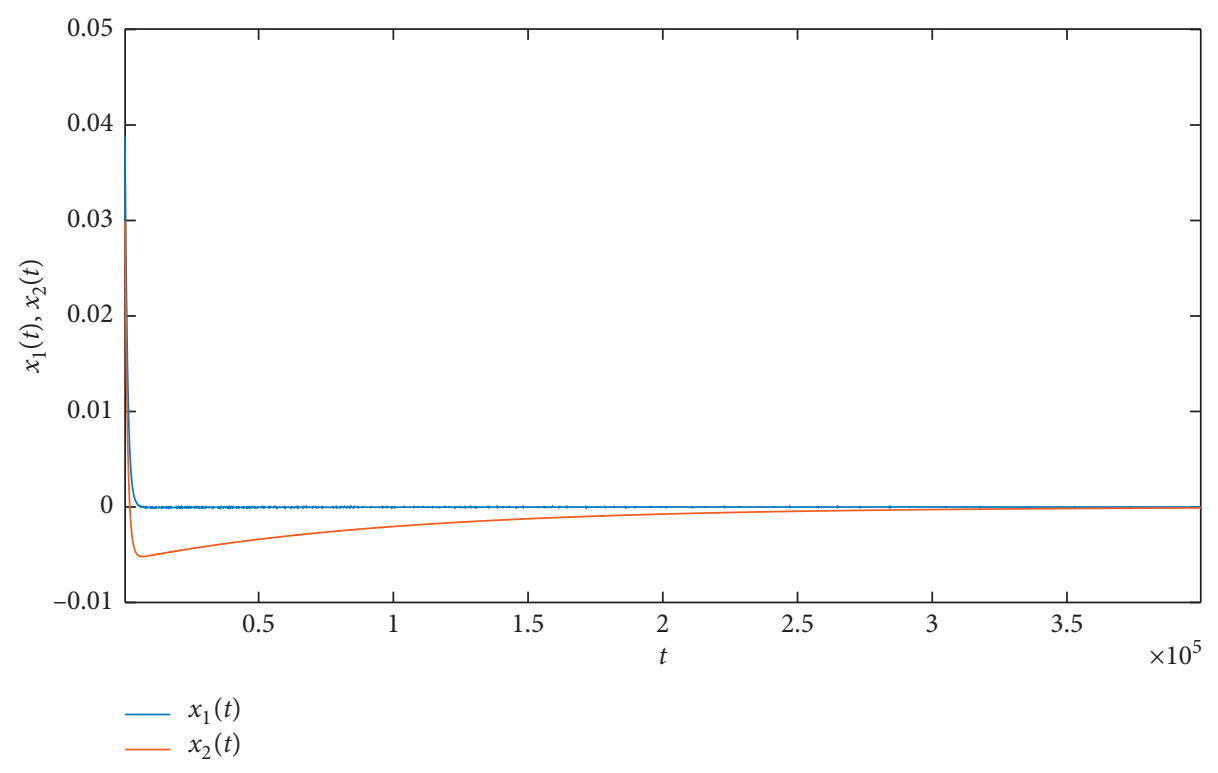

Figure 2: Time evolutions $x_{1}(t), x_{2}(t)$ of system (39) when $I_{1}, I_{2}=0$.

\section{Conclusions}

Memristors can show synaptic dynamic characteristic of the human brain, so it can become ideal implementation of the artificial synapse. Based on the memristors, some theoretical researchers look forward to propose some better model for understanding and simulating the characteristic of the human brain. In this paper, based on nonsmooth analysis, set-valued maps, and differential inequality analysis, we investigate input-tostate stability of a class of memristive simplified Cohen-Grossberg bidirectional associative memory (BAM) neural networks with variable time delays. Some sufficient conditions guarantee the input-to-state stability of such networks. Furthermore, when the input is zero, the stability of the total system is state stable. Our results extended to some known results, which can be applied in wider situations $[6,8,24-26]$. At the same time, an illustrative example shows the feasibility and effectiveness of our results.

\section{Data Availability}

No data were used to analyse this theoretical study.

\section{Conflicts of Interest}

The authors declare that there are no conflicts of interest regarding the publication of this paper.

\section{Acknowledgments}

This work was supported by the National Natural Science Foundation of China (nos. 11502073 and 11972115) and also partially supported by the Doctoral Foundation of Henan Polytechnic University (B2012-107).

\section{References}

[1] B. Kosko, "Bidirectional associative memories," IEEE Transactions on Systems, Man, and Cybernetics, vol. 18, no. 1, pp. 49-60, 1988.
[2] K. Gopalsamy and X. Z. He, "Delay-independent stability in bidirectional associative memory networks," IEEE Transactions on Neural Networks, vol. 5, no. 6, pp. 998-1002, 1994.

[3] J. D. Cao and L. Wang, "Exponential stability and periodic oscillatory solution in BAM networks with delays," IEEE Transactions on Neural Networks, vol. 13, pp. 457-463, 2002.

[4] Y. Li and C. Li, "Matrix measure strategies for stabilization and synchronization of delayed BAM neural networks," Nonlinear Dynamics, vol. 84, no. 3, pp. 1759-1770, 2016.

[5] J. Thipcha and P. Niamsup, "New exponential passivity of BAM neural networks with time-varying delays," Neural Computing and Applications, vol. 29, no. 12, pp. 1593-1600, 2018.

[6] W. G. Yang, W. W. Yu, and J. D. Cao, "Global exponential stability of impulsive fuzzy high-order BAM neural networks with continuously distributed delays," IEEE Transactions on Neural Networks and Learning Systems, vol. 29, pp. 36823700, 2018

[7] C. J. Xu, P. L. Li, and Y. C. Pang, "Existence and exponential stability of almost periodic solutions for neutral-type BAM neural networks with distributed leakage delays," Mathematical Methods in the Applied Sciences, vol. 40, pp. 21772196, 2017.

[8] Y. Zhao, Y. Xia, and W. Ding, "Periodic oscillation for BAM neural networks with impulses," Journal of Applied Mathematics and Computing, vol. 28, no. 1-2, pp. 405-423, 2008.

[9] X. He, T. W. Huang, J. Z. Yu, C. D. Li, and C. J. Li, "An inertial projection neural network for solving variational inequalities," IEEE Transactions on Cybernetics, vol. 47, no. 3, pp. 809-814, 2017.

[10] X. He, C. D. Li, T. W. Huang, C. J. Li, and J. J. Huang, "A recurrent neural network for solving bilevel linear programming problem," IEEE Transactions on Neural Networks and Learning Systems, vol. 25, no. 4, pp. 824-830, 2014.

[11] C. Y. Chen, S. Zhu, Y. C. Wei, and C. Y. Yang, "Finite-time stability of delayed memristor-based fractional-order neural networks," IEEE Transactions on Cybernetics, pp. 1-10, 2018.

[12] S. Zhu and Y. Shen, "Two algebraic criteria for input-to-state stability of recurrent neural networks with time-varying delays," Neural Computing and Applications, vol. 22, no. 6, pp. 1163-1169, 2013. 
[13] Y. Zhao, Q. Lu, and Z. Feng, "Stability for the mix-delayed cohen-grossberg neural networks with nonlinear impulse," Journal of Systems Science and Complexity, vol. 23, no. 3, pp. 665-680, 2010.

[14] C. Zhang, F. Deng, Y. Peng, and B. Zhang, "Adaptive synchronization of Cohen-Grossberg neural network with mixed time-varying delays and stochastic perturbation," Applied Mathematics and Computation, vol. 269, pp. 792-801, 2015.

[15] M. Zheng, L. Li, H. Peng, J. Xiao, Y. Yang, and H. Zhao, "Finite-time stability and synchronization for memristorbased fractional-order Cohen-Grossberg neural network," The European Physical Journal B, vol. 89, no. 9, p. 204, 2016.

[16] H. Bao, "Existence and exponential stability of periodic solution for BAM fuzzy Cohen-Grossberg neural networks with mixed delays," Neural Processing Letters, vol. 43, no. 3, pp. 871-885, 2016.

[17] R. Rao, S. Zhong, and Z. Pu, "On the role of diffusion factors in stability analysis for p-laplace dynamical equations involved to BAM Cohen-Grossberg neural network," Neurocomputing, vol. 223, pp. 54-62, 2017.

[18] R. Chinnathambi, F. A. Rihan, and S. Lakshmanan, "Stabilization of delayed Cohen-Grossberg BAM neural networks," Mathematical Methods in the Applied Sciences, vol. 41, pp. 593-605, 2018.

[19] L. Chua, "Memristor-the missing circuit element," IEEE Transactions on Circuit Theory, vol. 18, no. 5, pp. 507-519, 1971.

[20] D. B. Strukov, G. S. Snider, D. R. Stewart, and R. S. Williams, "The missing memristor found," Nature, vol. 453, no. 7191, pp. 80-83, 2008.

[21] R. Legenstein, "Nanoscale connections for brain-like circuits," Nature, vol. 521, no. 7550, pp. 37-38, 2015.

[22] L. Chen, C. Li, T. Huang, H. G. Ahmad, and Y. Chen, "A phenomenological memristor model for short-term/longterm memory," Physics Letters A, vol. 378, no. 40, pp. 29242930, 2014.

[23] L. Chen, C. Li, X. Wang, and S. Duan, "Associate learning and correcting in a memristive neural network," Neural Computing and Applications, vol. 22, no. 6, pp. 1071-1076, 2013.

[24] M. Itoh and L. O. Chua, "Memristor cellular automata and memristor discrete-time cellular neural networks," International Journal of Bifurcation and Chaos, vol. 19, no. 11, pp. 3605-3656, 2009.

[25] F. Corinto, A. Ascoli, and M. Gilli, "Nonlinear dynamics of memristor oscillators," IEEE Transactions on Circuits and Systems I: Regular Papers, vol. 58, no. 6, pp. 1323-1336, 2011.

[26] X. Yang, J. Cao, and W. Yu, "Exponential synchronization of memristive Cohen-Grossberg neural networks with mixed delays," Cognitive Neurodynamics, vol. 8, no. 3, pp. 239-249, 2014.

[27] A. Wu and Z. Zeng, "Lagrange stability of memristive neural networks with discrete and distributed delays," IEEE Transactions on Neural Networks and Learning Systems, vol. 25, no. 4, pp. 690-703, 2014.

[28] Y. Zhao, J. Kurths, and L. Duan, "Input-to-State stability analysis for memristive Cohen-Grossberg-type neural networks with variable time delays," Chaos, Solitons \& Fractals, vol. 114, pp. 364-369, 2018.

[29] K. Zhong, Q. Yang, and S. Zhu, "New algebraic conditions for ISS of memristive neural networks with variable delays," Neural Computing and Applications, vol. 28, no. 8, pp. 2089-2097, 2017.

[30] Y. Zhao, J. Kurths, and L. Duan, "Input-to-state stability analysis for memristive BAM neural networks with variable time delays," Physics Letters A, vol. 383, no. 11, pp. 1143-1150, 2019.

[31] C. Xu and P. Li, "pth moment exponential stability of stochastic fuzzy Cohen-Grossberg neural networks with discrete and distributed delays," Nonlinear Analysis: Modelling and Control, vol. 22, no. 4, pp. 531-544, 2017.

[32] C. $\mathrm{Xu}$ and P. Li, "Periodic dynamics for memristor-based bidirectional associative memory neural networks with leakage delays and time-varying delays," International Journal of Control, Automation and Systems, vol. 16, no. 2, pp. 535549, 2018.

[33] S. Zhu, D. Liu, C. Y. Yang, and J. Fu, "Synchronization of memristive complex-valued neural networks with time delays via pinning control method," IEEE Transactions on Cybernetics, pp. 1-10, 2019.

[34] D. Liu, S. Zhu, and K. Sun, "Global anti-synchronization of complex-valued memristive neural networks with time delays," IEEE Transactions on Cybernetics, vol. 49, no. 5, pp. 1735-1747, 2019.

[35] A. F. Filippov, Differential Equations with Discontinuous Right-Hand Sides, Kluwer, Dordrecht, Netherlands, 1988.

[36] J. P. Aubin and A. Cellina, Differential Inclusions, Springer, Berlin, Germany, 1984.

[37] H. K. Khalil, Nonlinear Systems, Prentice-Hall, Upper Saddle River, NJ, USA, 3rd edition, 2012. 